\title{
Die Digitale Mozart-Edition (DME)
}

\author{
INTERdisZIPLINÄRE PROJEKTE UNTER EINEM DACH
}

Mirijam Beier \& Till Reininghaus

Internationale Stiftung Mozarteum, Salzburg

beier@mozarteum.at, reininghaus@mozarteum.at

https://mozarteum.at/

Keywords: Music Encoding Initiative (MEI), Text Encoding Initiative (TEI), Digital Edition, Interdisciplinarity, Wolfgang Amadé Mozart

\begin{abstract}
The Digital Mozart Edition unites various, interdisciplinary and freely accessible projects under one roof. All subprojects, like DME::Letters \& Documents or DME::Music, aim at ensuring a versatile and long-term reuse and therefore are based on XML formats like TEI and MEI.
\end{abstract}

Die Digitale Mozart-Edition (DME) ist ein an der Internationalen Stiftung Mozarteum (ISM) in Salzburg angesiedeltes Langzeitprojekt, das dort gemeinsam mit dem in Los Altos, Kalifornien/USA, beheimateten Packard Humanities Institute (PHI) durchgeführt wird. Unter dem Dach der DME sind verschiedene Teilprojekte versammelt, deren gemeinsames Ziel es ist, das gesamte Schaffen des Komponisten Wolfgang Amadé Mozart (1756-1791) in seinen vielfältigen Facetten digital aufzubereiten und im Internet für nicht-kommerzielle Zwecke unentgeltlich zur Verfügung zu stellen. ${ }^{1}$ Hierdurch sollen Ergebnisse der Forschung einem möglichst großen Kreis von Interessierten, z.B. Wissenschaftler*innen, Musiker*innen oder Lehrer*innen, aber auch Mozart-Liebhaber*innen, zugänglich gemacht werden. Um eine vielfältige und langfristige Nachnutzung zu ermöglichen, verwendet die DME XMLbasierte Formate wie TEI und MEI.

Im Projekt DME::Librettos \& Texts bietet die DME diverse Editionen von historischen Publikationen, die mit Wolfgang Amadé Mozart und seiner Familie in Verbindung stehen. Vollständig liegt bereits die Edition sämtlicher Mozart-Libretti vor. ${ }^{2}$ Als erste kritische Online-Edition der Texte der 25 musikdramatischen Werke Mozarts schließt sie eine wichtige Lücke in der Mozart-Philologie. Dabei wurde von jedem Werk der von Mozart vertonte Text und der des originalen, zur Uraufführung gedruckten Librettos, teilweise auch der literarischen Vorlagen ediert. Die Online-Edition der Mozart-Libretti nutzt die Möglichkeiten der digitalen Anzeige, beispielsweise indem neben dem edierten Text auch die Lesarten der Quellen parallel angezeigt werden können. Interdisziplinär ist dieses Projekt der DME aufgrund

1 Vgl. das interne Memorandum of Understanding zwischen PHI und der ISM.

2 Internationale Stiftung Mozarteum, Salzburg, Mozart-Libretti - Online-Edition, Salzburg 2007ff., online unter: https://dme. mozarteum.at/text-editions/librettos/ (11.03.2019); vgl. auch lacopo CIVIDINI, Von der literarischen Vorlage zum vertonten Text. Fünf Schritte zu einer digitalen textgenetischen Edition von Operntexten am Beispiel von Wolfgang Amadé Mozarts Die Entführung aus dem Serail KV 384, in: editio 31 (2017), 79-89. 
seiner Verbindung zwischen editorischen Interessen der Germanistik, Romanistik und Musikwissenschaft. Exemplarisch deutlich wird dies anhand der phonetisch-metrischen Analyse der von Mozart vertonten italienischen Libretti. Sie bietet ein dynamisches Instrumentarium, um die komplexe Beziehung zwischen metrischem Duktus der Textvorlage und dessen musikalischer Umsetzung näher erforschen zu können. Darüber hinaus befinden sich zwei weitere Projekte im Rahmen von DME::Librettos \& Texts in Vorbereitung: Das Projekt Nissen Online sieht eine kritische Edition der ersten umfangreichen Mozart-Biographie vor, die der dänische Diplomat und zweite Ehemann Constanze Mozarts, Georg Nikolaus Nissen, zusammengestellt hatte und die 1829 im Verlag Breitkopf \& Härtel erstmals erschienen war. ${ }^{3}$ Sie legt die bislang nur in Ansätzen bekannten, äußerst vielschichtigen Quellen offen, auf denen die Biographie beruht, kommentiert sie und verlinkt die diversen Primär- und Sekundärquellen mit digitalen Ressourcen. Ebenfalls wird derzeit das Projekt Leopold Mozart Violinschule - Digitale Edition vorbereitet. ${ }^{4}$ Vorgesehen ist anlässlich des 300. Geburtstags von Leopold Mozart im Jahr 2019 eine kritische digitale Edition von dessen Violinschule online vorzulegen. In einem ersten Schritt wird sie die ersten drei deutschen Auflagen des Lehrwerks umfassen; später sollen diese durch die historische niederländische und französische Übersetzung der Violinschule ergänzt werden.

Ebenfalls die Ansprüche einer volldigitalen Edition erfüllt das Projekt DME::Letters \& Documents mit einer Online-Publikation von Briefen der Mozart-Familie sowie von Dokumenten zur Familien- und Stiftungsgeschichte. ${ }^{5}$ So sind über dieses Projekt z.B. sämtliche Briefe Wolfgang Amadé Mozarts in einer diplomatischen Transkription verfügbar. Soweit dies rechtlich möglich ist, wird parallel auch ein Bild des Briefes angeboten, um Transparenz zwischen der Transkription und der Originalhandschrift herzustellen. Im Zentrum stehen die Bestände an Briefen und Dokumenten der ISM als weltweit größte Sammlung von Schriftstücken aus der Mozart-Familie, wobei die Briefe und Dokumente Wolfgang Amadé Mozarts und seines Vaters Leopold den überwiegenden Anteil ausmachen.

Als Kernprojekt der DME ist die Musikedition DME::Music zu bezeichnen. ${ }^{6}$ Dazu gehört zunächst das erste Projekt der DME: die bereits 2006 veröffentlichte NMA Online. Diese bietet Nutzer*innen die Möglichkeit, sämtliche 26.000 Seiten der gedruckten Bände der gesamten, über mehr als 50 Jahre erarbeiteten Neuen Mozart-Ausgabe (NMA) online als PDF-Scans abzurufen. Neben den Vorworten, Notenseiten und Anhängen der einzelnen Bände kann direkt auch auf die entsprechenden Kritischen Berichte zugegriffen werden. Außerdem wurden beispielsweise englischsprachige Vorworte ergänzt. Wenn in den Kritischen Berichten, die in vielen Fällen erst einige Zeit nach dem Notenband erschienen sind, für bestimmte Takte Berichtigungen oder Ergänzungen vermerkt wurden, lassen sich diese durch einen Link im Notentext unmittelbar aufrufen. Zusätzlich sind die einzelnen Werke mit den Aufnahmen der Mozart Ton- und Filmsammlung verknüpft, sodass Nutzer*innen sich einen Überblick über die vorhandenen Aufnahmen verschaffen können. Dabei stellt die NMA Online von jedem Mozart-Werk mindestens eine Einspielung zur Verfügung, die direkt über die Website abgerufen werden kann. So können Partiturverlauf und Einspielung parallel verfolgt werden. DME::Music bietet auf diese Weise eine weitere wichtige Zugangsebene für die Auseinandersetzung mit Mozarts Musik.

3 Internationale Stiftung Mozarteum, Salzburg, Nissen Online, Salzburg 2014ff., online unter: https://dme.mozarteum.at/ text-editions/nissen-online/ (11.03.2019).

4 Internationale Stiftung Mozarteum, Salzburg, Leopold Mozart Violinschule - Digitale Edition, Salzburg 2018ff., online unter: https://dme.mozarteum.at/text-editions/leopold-mozart-violinschule/ (11.03.2019).

5 Internationale Stiftung Mozarteum, Salzburg, Mozart Briefe und Dokumente - Online-Edition, Salzburg 2007ff., online unter: https://dme.mozarteum.at/briefe-dokumente/ (11.03.2019).

6 Internationale Stiftung Mozarteum, Salzburg, DME::Music, Salzburg 2014ff., online unter: https://dme.mozarteum.at/ musik/ (11.03.2019). 
Bei der NMA Online handelt es sich um eine bildbasierte Online-Präsentation, die also noch nicht, wie beschrieben, die Ansprüche einer genuin digitalen Edition erfüllt. Dieser erste digitale Schritt verfolgt primär das Ziel, den Zugriff auf die historisch-kritische Gesamtausgabe der Werke Wolfgang Amadé Mozarts zu vereinfachen und für Interessierte einen uneingeschränkten Zugang zu gewährleisten.

Als Nachfolgeprojekt der NMA Online versteht sich die Digital Interactive Mozart Edition (DIME) ${ }^{7}$, die auf den wissenschaftlichen Ergebnissen der NMA basiert, mit dieser aber nicht identisch ist und diese auch nicht ersetzt. DIME ist im Gegensatz zur NMA Online eine genuin digitale Edition, die mit dem XML-basierten Format der Music Encoding Initiative (MEI) arbeitet. Sie ermöglicht es, die Codierung mit weiteren Informationen anzureichern, die über den reinen Notentext hinausgehen, etwa zu editorischen Entscheidungen oder den Metadaten des entsprechenden Werkes. Hierdurch kann ein höherer Grad an Transparenz als bei gedruckten Editionen erreicht werden. Außerdem sind jederzeit Aktualisierungen möglich, die durch Vergabe von Versionsnummern nachvollzogen werden können. Dabei verfolgt DIME zwei editorische Stränge: Auf der einen Seite wird der gesamte Notentext der gedruckten NMA-Bände in sogenannten Referenztexten entsprechend in MEI codiert und angereichert. Die Basis hierfür bildet die Konvertierung des vollständigen Notentexts der NMA in ein MEl-konformes XML-File, das im Folgenden intensiv Korrektur gelesen, nach Richtlinien bearbeitet und um zusätzliche Informationen ergänzt wird, bevor die einzelnen Werke sukzessiv online geschaltet werden. Auf der anderen Seite werden ausgewählte Werke im Rahmen von sogenannten Alternativtexten neu ediert. Dabei handelt es sich um Quelleneditionen, die bereits vorhandene Referenztexte ergänzen.

Die entsprechend in MEI codierten Notentexte werden in einer eigens entwickelten Webanwendung, dem Digital Mozart score Viewer (MoVi), visualisiert, der wiederum auf dem durch RISM-Schweiz entwickelten Visualisierungstool Verovio fußt. ${ }^{8}$ Die diversen Features von MoVi werden ständig erweitert und fortentwickelt. In der folgenden Übersicht werden deshalb diejenigen Möglichkeiten der Benutzung und Werkzeuge vorgestellt, die beim First Release des MoVi am 14. Dezember 2018 implementiert waren (vgl. Abb. 1).

Unterhalb des Fensters, in dem der Notentext angezeigt wird, befinden sich verschiedene Schaltflächen, die der Navigation innerhalb des Notentextes dienen. So kann geblättert, durch Eingabe einer bestimmten Takt- oder Seitenzahl diese direkt angewählt und über eine Zoom-Leiste die Größe des angezeigten Notentextes verändert werden. Durch das Aktivieren der "Original-Zeilenumbrüche“ kann die dynamische Anzeige des Notentextes ausgeschaltet und der NMA angeglichen werden. Die letztgenannte Funktion ist insbesondere dann interessant, wenn ein Referenz- oder Alternativtext mit dem entspechenden Scan aus der NMA Online verglichen werden soll, was durch Klicken auf die Schaltfläche „Notentext in der NMA Online ansehen“ in der rechten Menüspalte unter „Übersicht“ im Splitscreen problemlos möglich ist. Ebenfalls unter dem Menüpunkt „Übersicht“ lassen sich diejenigen Werke, die bereits im Internet zur Verfügung stehen, über die „Werksuche“ nach KV-Nummern auswählen. Nach der Auswahl werden die einzelnen Sätze des Werks angezeigt. Außerdem kann hier falls vorhanden - zu dem entsprechenden Alternativtext, etwa der Quellenedition nach Autograph oder

7 Internationale Stiftung Mozarteum, Salzburg, Digital Interactive Mozart Edition, Salzburg 2018ff., online unter: https://dme. mozarteum.at/musik/edition/ (11.03.2019); vgl. auch Norbert DuBowy, Bereitstellung und Nutzung von Daten in der Digitalen Mozart Edition, in: Klaus Pietschmann, Hg., Symposiumsbericht „Wege des Faches - Wege der Forschung?“, Mainz 2018, 1-6, URN: nbn:de:101:1-2018122012143896934537.

8 RISM-Schweiz, Verovio. A music notation engraving library, [o. O. o. J.], online unter: https://www.verovio.org/index.xhtml (11.03.2019). 
Erstdruck, gewechselt werden. Mit dem Release wurden die sechs sogenannten Haydn-Quartette (KV 387, 421, 428, 458, 464 und 465), die Serenade in G Eine kleine Nachtmusik KV 525, das Ave verum corpus KV 618 und das Rondo der Vitellia "Non più di fiori“ aus La clemenza di Tito KV 621 als Referenztexte publiziert. Darüber hinaus wurden als Quelleneditionen das Streichquartett in B KV 458 vollständig nach dem Erstdruck und der erste Satz nach dem Autograph im Rahmen der Alternativtexte vorgelegt.

\section{MoVi The digital Mozart score Viewer}

\section{$? \equiv$}

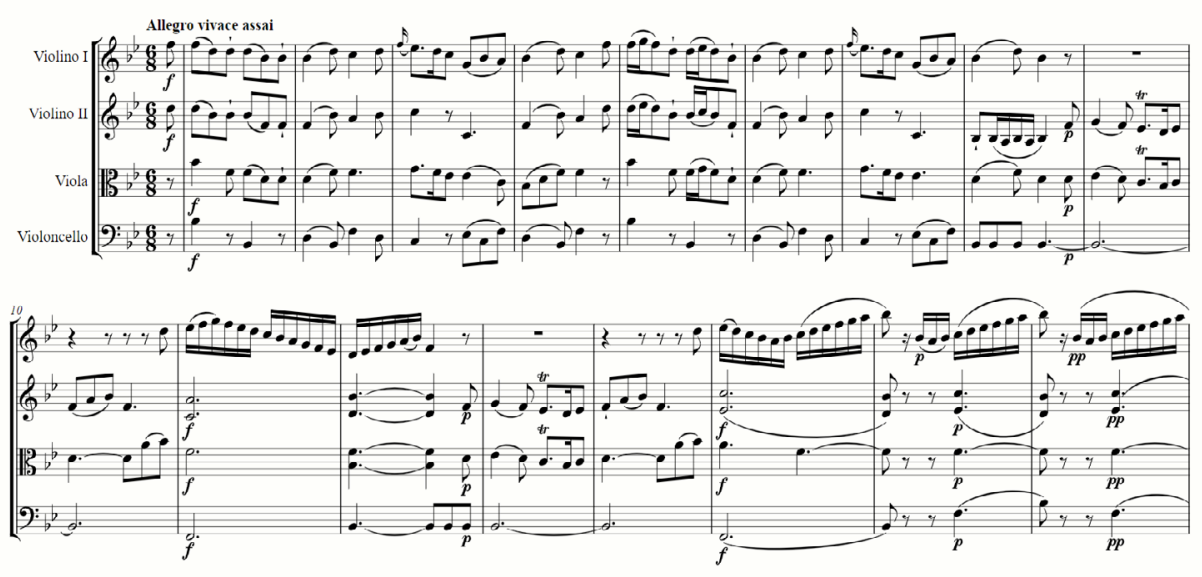

JBERSICHT • DASHBOARD • MEICODE

Quartett in B für zwei Violinen, Viola und Violoncello KV 458 NMA digital

1. Satz

Allegro vivace assai

Notentertinder NMA Orline anselen

Wählen Sie einen anderen Teil dieses Werkes aus

1: Allegro vivace assal

3:Adagio

Wählen Sie eine andere Repräsentation dieses Werkes aus:

KV 158 Autograph

KV 458 Erstdruck

\section{(14) (1) (1) 에 [seter 1 von 16]}
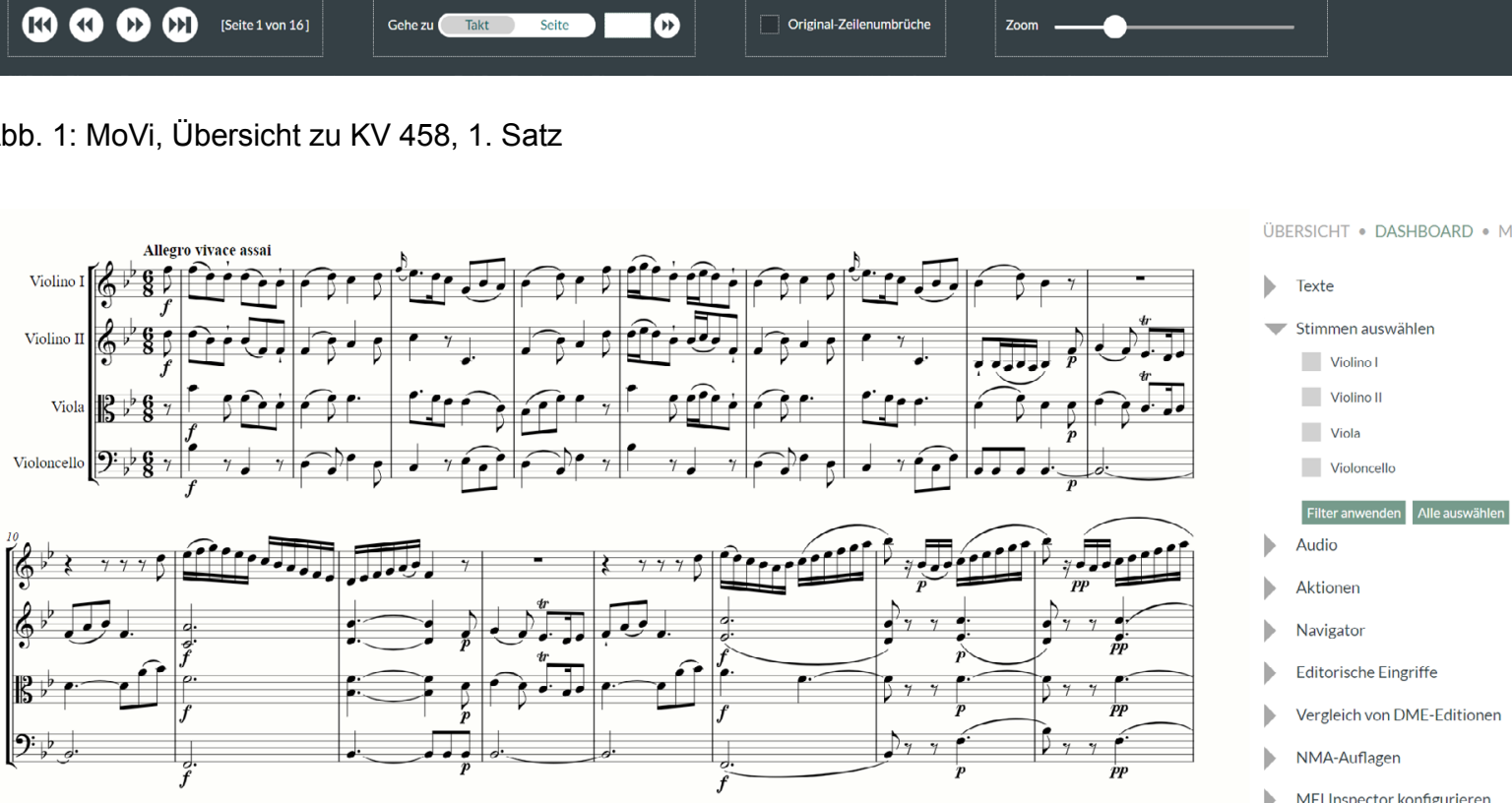

\section{(14) (1) (1)

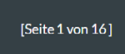

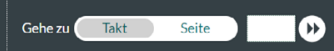

Original-Zeilenumbrüche

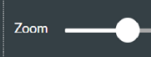

Abb. 2: MoVi, Dashboard

Wechselt man in der rechten Menüspalte von „Übersicht“ zu „Dashboard“, lassen sich verschiedene Werkzeuge auswählen. Hierunter finden sich praktische Tools, die etwa die Möglichkeit bieten, sich nur einzelne Stimmen anzeigen zu lassen, oder unter „Aktionen“ eine Funktion zum PDF-Druck des Noten- 
textes. Auch steht hier das gesamte File als Download sowie die Metadaten im Dublin Core-Format ${ }^{9}$ zur Verfügung. Der gesamte Webauftritt der DME steht unter der Lizenz CC BY-SA NC 4.010. Weitergabe und Bearbeitung der Files sind demnach unter bestimmten Bedingungen für nicht-kommerzielle Zwecke erlaubt. Der codierte Notentext kann im MIDI-Format abgespielt werden, für manche Werke steht auch eine Audio-Synchronisation mit mindestens einer Einspielung zur Verfügung. Unabhängig vom gewählten Abspielmodus wird der zu hörende Takt farblich hinterlegt, am Seitenumbruch blättert MoVi automatisch zur folgenden Seite um. Nutzer*innen können über den „MEl-Inspector“ auswählen, ob beim Klicken in den Notentext der MEI-Code eines einzelnen Elements, einer Stimme oder eines ganzen Taktes angezeigt werden soll. Der „Navigator“ dient der Zitation. Verwiesen werden kann mit inm auf ein gesamtes Werk, einen Satz daraus oder - noch detaillierter - auf einen bestimmten Takt bzw. eine Stimme in diesem Takt, indem eine URL erzeugt wird, mit der die ausgewählte Stelle präzise nachgewiesen werden kann. Philologisch untermauert wird DIME durch Texte zur Edition und Codierung, die einem Vorwort analoger Notenausgaben entsprechen. Bei Alternativtexten sind Quellenbeschreibungen und -bewertungen Teil des editorischen Angebots. Außerdem finden sich hier Schaltflächen, mit denen editorische Eingriffe im Notentext farblich hervorgehoben werden. Hier können gegebenenfalls auch Unterschiede zwischen NMA-Auflagen angezeigt oder die Differenzen zwischen den diversen Editionen von DIME (Referenztext vs. Alternativtext) visualisiert werden. Im Fall der Alternativtexte besteht außerdem die Möglichkeit, Annotationen der Editor*innen einzublenden. Augenfällig wird der Zusammenhang von Visualisierung und Codierung, wenn in der rechten Menü-Spalte „MEl Code“ ausgewählt wird: Dies hat zur Folge, dass der Code neben dem Notenbild sichtbar wird, wobei ein entweder im Code oder im Notentext angewähltes Element in den beiden Darstellungsformen jeweils hervorgehoben und somit eine Verbindung zwischen beiden geschaffen wird.

MoVi The digital Mozart score Viewer

DE $? \equiv$

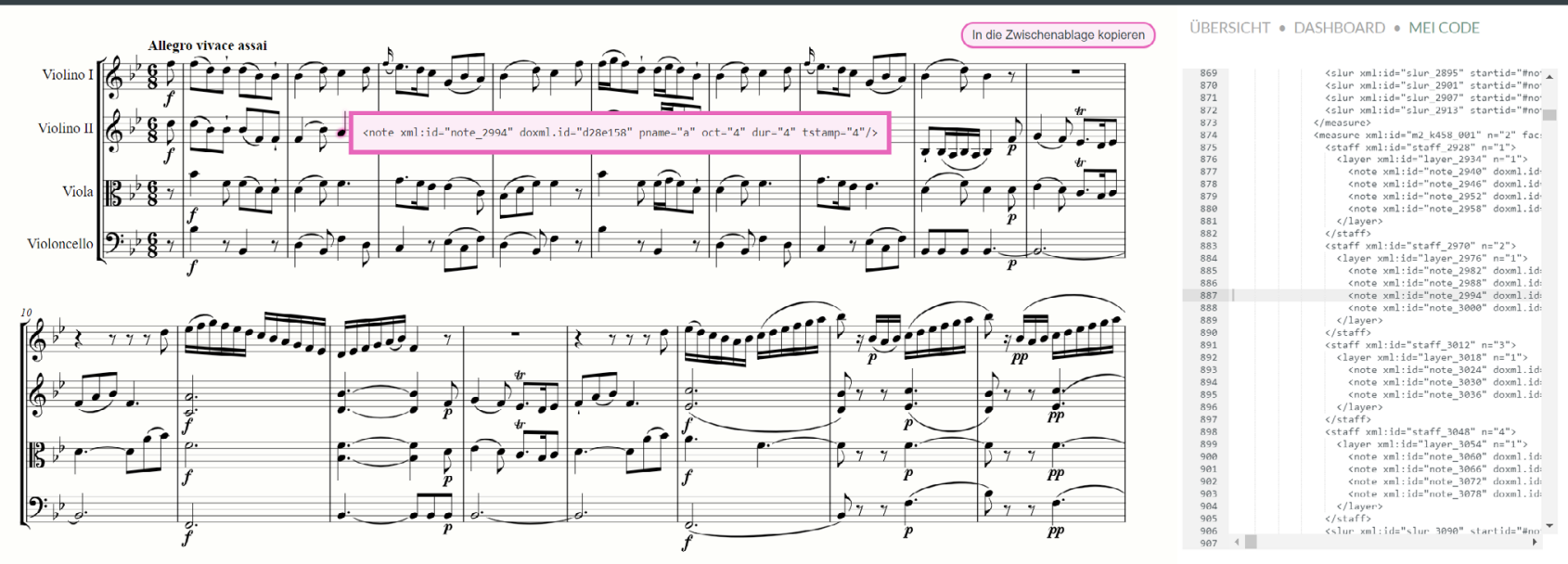

\section{$(14)(1)$}

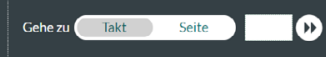

OriginalZzeilenumbrichehe

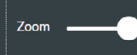

Abb. 3: MoVi, Verbindung Notentext - MEl Code

9 N. N., Dublin Core Metadata Initiative, [o. O.] 1995-2019, online unter: http://dublincore.org/ (11.03.2019).

10 Creative Commons, Namensnennung - Weitergabe unter gleichen Bedingungen 4.0 International (CC BY-SA 4.0), Mountain View, California (USA) [o. J.], online unter: https://creativecommons.org/licenses/by-sa/4.0/deed.de (11.03.2019). 
In Zukunft werden die Angebote der DIME regelmäßig erweitert und aktualisiert: Monatlich kommen im Bereich der Referenztexte neue Werke hinzu. Auch das Angebot der Alternativtexte soll stetig erweitert werden. Durchaus der Philosophie der DME entspricht es, dass Nutzer*innen die MEI-Codierung weiternutzen, indem sie die von DIME zur Verfügung gestellten Daten in eigene Projekte integrieren. Das daraus entstehende Potenzial scheint vielversprechend. Mit dem Umfang und Anspruch der diversen Projekte unter dem Dach der DME bricht die Mozart-Editorik in ein neues Zeitalter auf.

\section{Literaturverzeichnis}

lacopo CIVIDINI, Von der literarischen Vorlage zum vertonten Text. Fünf Schritte zu einer digitalen textgenetischen Edition von Operntexten am Beispiel von Wolfgang Amadé Mozarts Die Entführung aus dem Serail KV 384, in: editio 31 (2017), 79-89.

Creative Commons, Namensnennung - Weitergabe unter gleichen Bedingungen 4.0 International (CC BY-SA 4.0), Mountain View, California (USA) [o. J.], online unter: https://creativecommons.org/licenses/by-sa/4.0/ deed.de (11.03.2019).

Norbert DUBOWY, Bereitstellung und Nutzung von Daten in der Digitalen Mozart Edition, in: Klaus Pietschmann, Hg., Symposiumsbericht „Wege des Faches - Wege der Forschung?“, Mainz 2018, 1-6, URN: nbn:de:101:1-2018122012143896934537.

Internationale Stiftung Mozarteum, Salzburg, Digital Interactive Mozart Edition, Salzburg 2018ff., online unter: https://dme.mozarteum.at/musik/edition/ (11.03.2019).

Internationale Stiftung Mozarteum, Salzburg, Leopold Mozart Violinschule - Digitale Edition, Salzburg 2018ff., online unter: https://dme.mozarteum.at/text-editions/leopold-mozart-violinschule/ (11.03.2019).

Internationale Stiftung Mozarteum, Salzburg, Mozart Briefe und Dokumente - Online-Edition, Salzburg 2007ff., online unter: https://dme.mozarteum.at/briefe-dokumente/ (11.03.2019).

Internationale Stiftung Mozarteum, Salzburg, Mozart-Libretti - Online-Edition, Salzburg 2007ff., online unter: https://dme.mozarteum.at/text-editions/librettos/ (11.03.2019).

Internationale Stiftung Mozarteum, Salzburg, Nissen Online, Salzburg 2014ff., online unter: https://dme. mozarteum.at/text-editions/nissen-online/ (11.03.2019).

N. N., Dublin Core Metadata Initiative, [o. O.] 1995-2019, online unter: http://dublincore.org/ (11.03.2019).

RISM-Schweiz, Verovio. A music notation engraving library, [o. O., o. J.], online unter: https://www.verovio.org/ index.xhtml (11.03.2019). 